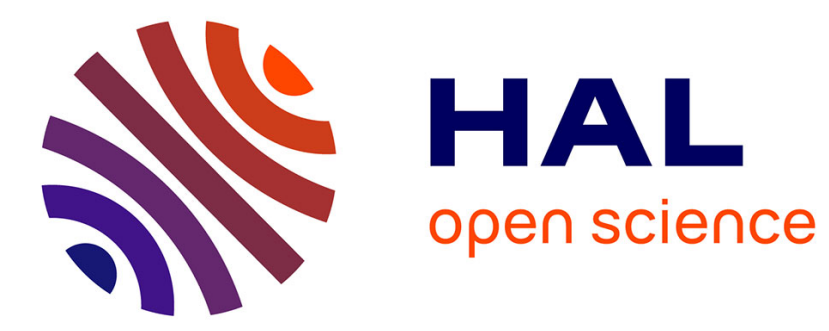

\title{
Une agence au service d'une stratégie ministérielle
}

Matthieu Ansaloni, Andy Smith

\section{To cite this version:}

Matthieu Ansaloni, Andy Smith. Une agence au service d'une stratégie ministérielle. Gouvernement $\&$ action publique, 2018, 7 (1), pp.33-55. 10.3917/gap.181.0033 . halshs-02111572

\section{HAL Id: halshs-02111572 \\ https://shs.hal.science/halshs-02111572}

Submitted on 26 Apr 2019

HAL is a multi-disciplinary open access archive for the deposit and dissemination of scientific research documents, whether they are published or not. The documents may come from teaching and research institutions in France or abroad, or from public or private research centers.
L'archive ouverte pluridisciplinaire HAL, est destinée au dépôt et à la diffusion de documents scientifiques de niveau recherche, publiés ou non, émanant des établissements d'enseignement et de recherche français ou étrangers, des laboratoires publics ou privés.

$$
\text { Copyright }
$$




\section{UNE AGENCE AU SERVICE D'UNE STRATÉGIE MINISTÉRIELLE La crise du Mediator et la concordance des champs \\ Matthieu Ansaloni et Andy Smith}

Presses de Sciences Po | «Gouvernement et action publique »

$2018 / 1 \mathrm{~N}^{\circ} 1 \mid$ pages 33 à 55

ISSN 2260-0965

ISBN 9782724634969

Article disponible en ligne à l'adresse :

https://www.cairn.info/revue-gouvernement-et-action-publique-2018-1-page-33.htm

Distribution électronique Cairn.info pour Presses de Sciences Po.

(C) Presses de Sciences Po. Tous droits réservés pour tous pays.

La reproduction ou représentation de cet article, notamment par photocopie, n'est autorisée que dans les limites des conditions générales d'utilisation du site ou, le cas échéant, des conditions générales de la licence souscrite par votre établissement. Toute autre reproduction ou représentation, en tout ou partie, sous quelque forme et de quelque manière que ce soit, est interdite sauf accord préalable et écrit de l'éditeur, en dehors des cas prévus par la législation en vigueur en France. Il est précisé que son stockage dans une base de données est également interdit. 


\title{
UNE AGENCE AU SERVICE D'UNE STRATÉGIE MINISTÉRIELLE
}

\section{La crise du Mediator et la concordance des champs}

\author{
Matthieu Ansaloni, Andy Smith
}

Résumé : À partir de l'exemple de la décision de suspension de médicaments antidiabétiques, cet article pose la question de la capacité d'action autonome des agences de réglementation vis-à-vis de leur autorité ministérielle. II montre que cette décision, prononcée formellement par l'Agence française de sécurité sanitaire des produits de santé (AFSSAPS), fut imposée, dans les faits, par le ministre de la Santé et ses conseillers. Faire cette démonstration conduit à apporter un démenti au lieu commun académique selon lequel l'« agencification » est au fondement d'un «État fort ». L'article fait apparaître les conditions structurales au sein desquelles se sont déployées les stratégies de divers agents qui ont suscité l'intervention ministérielle. Au-delà, il pose quelques jalons pour saisir sociologiquement la façon dont les moments critiques favorisent une concordance de logiques d'action de fractions appartenant à des champs distincts, établissant un rapport de force dominant.

MOTS-CLÉS : AGENCE - MINISTRE - SANTÉ

\section{AN AGENCY AT THE WHIM OF A MINISTER'S STRATEGY : THE MEDIATOR CRISIS AND THE CONCORDANCE OF FIELDS}

Abstract: On the basis of the example of the suspension of anti-diabetic medicines in France, this article addresses the question of regulatory agencies' autonomous capacity of action as regards their respective minister. The article shows that this decision of the Agence Française de Sécurité Sanitaire des Produits de Santé (AFSSAPS) was effectively imposed by the minister for Health and his close advisers. In demonstrating how and why this occurred, our analysis challenges an academic orthodoxy which considers agencization to be a foundation of a "strong state". More precisely, we reveal the structural conditions that enabled different actors to adopt strategies that, ultimately, rendered the ministerial intervention possible. More generally, we propose a means of sociologically studying the moments of contingency which favor concordance between the logics of action adopted by certain factions belonging to separate fields. We show how this convergence creates a dominant set of power relations.

KEYWORDS: AGENCY - HEALTH - MINISTER

9 juin 2011 : l'Agence française de sécurité sanitaire des produits de santé (AFSSAPS) se distingue. Son directeur, le professeur d'oncologie Dominique Maraninchi, annonce par 
un communiqué de presse la suspension des médicaments à base de pioglitazone Actos et Competact : la molécule, qui est administrée à quelque 230000 patients diabétiques, est suspectée de favoriser la survenue de cancers de la vessie. Le directeur de l'AFSSAPS rend publique la décision prise le jour même par la commission d'autorisation de mise en marché (AMM), l'instance centrale de l'agence, que ses experts ont voté à une très large majorité (25 voix pour, 1 abstention). Accessible sur le site internet de l'AFSSAPS, la séance donne à voir une agence qui agit en toute indépendance, prenant ses décisions sur la base des seules considérations scientifiques. D'abord, un évaluateur de l'agence en charge du dossier liste, publications scientifiques à l'appui, les bénéfices et les risques des médicaments à base de pioglitazone. Ensuite, une étude que l'AFSSAPS a commandée quelques mois auparavant à la Caisse nationale d'assurance maladie des travailleurs salariés (CNAM-TS) est présentée : ses résultats, qui s'appuient sur une cohorte de près d'un million et demi de personnes, indiquent que l'administration des médicaments à base de pioglitazone favorise une faible augmentation du risque de cancer de la vessie. Sur cette base, quelques échanges émergent entre les experts de la commission d'AMM. Le rapporteur du dossier semble cependant susciter d'emblée l'adhésion de la salle : l'étude de la CNAM-TS confirmerait un faisceau d'indices préexistant qui établissait une corrélation entre administration de médicaments à base de pioglitazone et survenue de cancers de la vessie. De plus, selon le rapporteur du dossier, les médicaments contre le diabète ne manquent pas : les alternatives thérapeutiques sont nombreuses ; les patients ne souffriront pas de la décision de suspension. L'évidence paraît s'imposer avec force aux experts de la commission : la balance entre les bénéfices et les risques des médicaments à base de pioglitazone est négative. Survient enfin le vote, écrasant, des experts qui composent la commission.

Cette décision est tout sauf anodine. En suspendant l'usage des médicaments à base de pioglitazone, le directeur de l'AFSSAPS engage un double bras de fer : avec les responsables de la firme japonaise Takeda qui commercialisent ces spécialités thérapeutiques; avec la Commission européenne qui, sur la base de l'avis de la European Medicines Agency (EMA), avait autorisé en 2000 leur mise sur le marché, une décision qui s'imposait aux États membres. Le 21 juillet 2011, les experts de l'EMA, qui avaient entrepris à l'initiative de l'AFSSAPS une réévaluation du rapport entre les bénéfices et les risques des médicaments à base de pioglitazone, maintiennent leur position. Juridiquement caduque, la décision de l'AFSSAPS ne sera cependant pas remise en cause. C'est donc un État héroïque qui est mis en scène : son bras armé - l'AFSSAPS - exerce son autorité de réglementation indépendamment, mobilisant - entre autres sources - une expertise publique que sa direction a commandée. Appuyé par son agence, l'État français s'oppose, au nom de la santé de ses administrés, au fleuron de l'industrie pharmaceutique japonaise et à la Commission européenne.

Disons-le d'emblée : les apparences - soigneusement entretenues par les responsables de l'AFSSAPS - sont trompeuses. En aucun cas, la décision de suspendre les médicaments à base de pioglitazone n'est le fruit d'une agence exerçant une autorité réglementaire sur la base de seules considérations scientifiques, à distance de la tutelle ministérielle, bien au contraire. Nous montrerons que cette décision est le fruit d'une action pilotée depuis le cabinet du ministre de la Santé qui vise, en restaurant la réputation d'une agence discréditée par ce qui s'impose alors dans les esprits comme "le scandale du Mediator ", à préserver 
- sinon augmenter - le capital symbolique du ministre. En faisant cette démonstration, cet article entend apporter une double plus-value :

- En rapport aux études sur l'« agencification » des États, il suggère une lecture structurale de l'action des agences : se distanciant de la fiction qui représente les agences comme des organisations « indépendantes ", notre lecture appréhende leur action comme l'expression de l'état du rapport de forces entre et à l'intérieur de différents champs sociaux ;

- En rapport à la théorie sociologique, cet article suggère quelques jalons pour saisir la façon dont les moments critiques (ici «le scandale du Mediator »), en favorisant un alignement des luttes propres à certains champs sociaux, alimentent une concordance de logiques d'action de fractions évoluant en leur sein, établissant un rapport de force dominant. Dit autrement, dans certaines conditions, l'alignement des luttes propres à des champs distincts qu'induit une situation de "crise » est susceptible de subvertir les hiérarchies établies".

\section{De l'« indépendance » des agences à leur encastrement social}

Pour faire apparaître cette double plus-value, il importe d'abord d'opérer un retour sur les études sur l'« agencification » des États, puis d'esquisser la base conceptuelle de notre perspective analytique. Nous pourrons dès lors préciser les mécanismes - entendus comme des processus qui engendrent des effets - qui sont à l'origine de la suspension des médicaments à base de pioglitazone. Les parties empiriques du présent article visent quant à elles à reconstituer la façon dont lesdits mécanismes opèrent empiriquement.

\section{L'« agencification » au fondement d'un État fort}

La création des agences - phénomène d'ordinaire perçu comme un fait marquant de la recomposition des États, généralement associé à leur «managérialisation » - a suscité une littérature importante. Une ligne d'analyse dominante se dégage : l'" agencification " serait au fondement d'un nouvel "État fort ". Le phénomène d'accumulation et de concentration de ressources (expertise notamment) qui accompagne ce processus serait à l'origine d'un mouvement nouveau de différenciation des États (Rose, Miller, 1992 ; Moran, 2002 ; Gilardi, 2005 ; Hanretty, Koop, 2012, 2013 ; Le Galès, 2014). L'“ agencification " - caractéristique pour les uns de l'État-régulateur (Majone, 1997 ; Moran, 2002), pour les autres du capitalisme-régulateur (Levi-Faur, 2005) - signerait l'avènement d'une élite nouvelle : la "régulocratie " (Levi-Faur, 2005 ; Gilardi, Maggetti, 2010). Les agences prendraient le pas sur les acteurs politiques traditionnels - groupes d'intérêt, administrations étatiques, élus politiques. Riches et nombreuses, les études des sociologues et des politistes sur l'"agencification " de l'administration de la Santé française - parmi laquelle compte l'Agence du médicament qui est au cœur de cet article - donnent dans une certaine mesure crédit à cette ligne

\footnotetext{
1. Nous remercions les évaluateurs de versions antérieures de ce texte dont les remarques nous ont conduites à identifier ce point avec plus de clarté. Notre reconnaissance va aussi à Aïcha Bourad. Frédéric Nicolas et Antoine Roger pour les commentaires qu'ils ont formulés sur ce travail.
} 
d'analyse 2 . Sorte d'incarnation de la doctrine de la sécurité sanitaire, produit intellectuel de quelques conseillers de Bernard Kouchner alors ministre de la Santé (Alam, 2010 ; Bergeron, Nathanson, 2012), les agences qui émergent crise après crise à partir des années 1990, constituent de nouvelles formes politiques qui s'apparentent à des «bureaucraties techniques » (Benamouzig, Besançon, 2005, 2008). À cheval entre espace scientifique et espace administratif, elles élargissent le "périmètre de l'administration » tout en renforçant ses "structures " (Benamouzig, Besançon, 2005, p. 301). Si le modèle de l'" expertise confinée " (Callon et al., 2001) qui caractérise leur action opacifie les procédures, elle est cependant gage de leur « indépendance », notamment vis-à-vis des responsables politiques, donc de leur " efficacité » (Benamouzig, Besançon, 2005, p. 301). Ce serait le cas de l'Agence française de sécurité sanitaire des aliments - AFSSA (Besançon, 2010 ; Granjou, Barbier, 2004) comme de l'Agence du médicament: celle-ci - constituée dans les discours experts et savants d'agence modèle en ce qu'elle dispose d'un pouvoir d'évaluation et de réglementation - marquerait pour Urfalino (2001) le renforcement de l'administration du Médicament, dans la mesure où elle dispose de moyens accrus (personnel notamment), en dépit - toujours selon le même auteur - des similarités frappantes qu'elle entretient avec la direction de la Pharmacie et du Médicament (DPHM) du ministère de la Santé ${ }^{3}$.

D'autres analyses nuancent ces résultats. Certes, pour Buton et Pierru, les agences sanitaires sont une manifestation du « renforcement de l'État sanitaire central » (2012, p. 68), une dimension organisationnelle qui s'appuie sur l'émergence d'une nouvelle élite administrative, dite "élite du Welfare» (Hassenteufel et al., 1999), dont une conséquence est le "renforcement de l'intégration institutionnelle " des professions médicales (Benamouzig, Pierru, 2011, p. 3). Cependant, pour Buton et Pierru, les agences constituent aussi des ressources pour les responsables politiques : I'expertise qui (dans les textes du moins) assoit leurs décisions neutralise celles-ci, favorisant leur acceptation; de plus, les agences sont des « fusibles " pour les dirigeants politiques quand leur responsabilité est mise en cause. Plus encore, comme l'a montré Buton (2006), l'action des agences - ici l'Institut national de veille sanitaire (INVS) - est une " activité indexée de manière continuelle, et non pas ponctuelle sur les préoccupations du politique » (p. 80). Instituée comme un savoir d'État au service de l'État, l'épidémiologie qui guide la production de l'expertise de l'INVS vise ainsi à offrir une réponse à une "situation politique problématique " (p. 84). En somme, pour Buton et Pierru, les agences sont certes à cheval entre les espaces administratif et scientifique, mais aussi partisan. Les effets de l'«agencification " - tantôt moteur du renforcement de l'État, tantôt moyen d'action pour les responsables politiques - sont équivoques. Cette perspective, que nous partageons pleinement, présente cependant deux inconnues: la

2. Nous mettons de côté la littérature internationale de science politique sur les agences: reposant sur des études comparatives basées sur un grand nombre de cas et de pays, celles-ci font l'impasse sur les contextes (les systèmes de position) et les dispositions des acteurs. De fait, la cumulativité de leurs résultats avec ceux issus des études inscrites dans la tradition sociologique n'est guère possible. Retenons que, validant les thèses des tenants du capitalisme-régulateur, elles tendent à apporter une caution scientifique aux discours officiels selon lesquels les agences - de par leur indépendance - sont gages d'une meilleure efficacité de l'action publique. Voir, pour une synthèse Maggetti, Verhoest (2014).

3. Similarités qui tiennent - ce n'est pas la moindre des choses - à son organisation interne : l'Agence du médicament, comme la plupart des agences françaises, repose sur le modèle dit de l'expertise externe. En clair, cette agence est dépourvue d'équipes de scientifiques (sauf quelques cadres en détachement), donc d'expertise propre; ses décisions sont prises avec l'appui d'experts extérieurs (universitaires, médecins). 
première concerne les rapports qu'entretiennent agences et responsables politiques; la seconde concerne les effets des crises - omniprésentes dans les analyses sur l'" agencification " de l'administration de la Santé - sur l'activité des agences. L'étude de cas dont nous rendons compte ici, qui prend à revers la doxa académique associant " agencification " et «État fort », apporte quelques éclairages en la matière.

\section{Saisir les agences en acte : une lecture structurale}

Inspirée de la théorie des champs de Bourdieu, notre perspective part des systèmes de position, c'est-à-dire des rapports de force qui structurent les champs. Appréhendant l'action publique - ici une décision - comme le fruit d'une alliance entre des fractions appartenant à des champs distincts (Bourdieu, 2000), notre démarche vise à saisir les relations qui se tissent dans et entre les champs au sein desquels est élaborée l'action publique. L'observation s'oriente vers l'identification des positions (dominants/dominés) des agents qui tissent ces relations, lesquelles découlent de leurs propriétés sociales que révèlent les sortes de capitaux dont ils disposent (Neveu, 2013). Notre démarche se distancie donc d'un usage monographique du concept de champ pour porter l'attention sur les relations qui se tissent certes en leur sein mais aussi entre eux (Dubois, 2014 ; Itçaina et al., 2016). Cependant, dans l'esprit de la théorie des champs, la compréhension desdites relations implique de resituer leurs conditions structurales, la position qu'occupent les agents en question, la logique de leur action (l'enjeu propre à leur champ), les capitaux dont ils disposent - saisis à travers leur histoire propre. Cet article porte l'attention sur des agents qui évoluent dans quatre champs : l'administration de la Santé (incluant les agences placées sous la tutelle du ministère) ; le champ partisan ; le champ des journalistes de la santé ; le champ scientifique.

Structurale, notre approche appréhende la production des alliances entre des agents évoluant dans des champs distincts comme le fruit de l'évolution de rapports de force qui leur sont internes: des fractions évoluant dans des champs distincts convergent vers une vision du monde commune en fonction des luttes qui leur sont propres (Bourdieu, 2000). L'action publique s'analyse ainsi, pour faire image, comme le fruit de "concordance de champs " (Dubois, 2014) ou de "coïncidences structurales " (Itçaina et al., 2016). Cette convergence n'est pas le fruit du hasard: elle est le résultat cumulatif de luttes politiques que déploient les agents pour faire valoir leur point de vue - définir une situation, les instruments pour la résoudre, asseoir leur bien-fondé. Ce travail politique par lequel émergent les pensées dominantes s'inscrit notamment dans des «lieux neutres ", des sortes de laboratoires idéologiques situés à l'intersection des champs administratif, scientifique et partisan (Bourdieu, 2000). Ils constituent des points d'entrée pour saisir la façon dont les pensées dominantes sont produites, tant par l'observation du travail idéologique qui s'y déploie que par celle de sa mise en scène. Les commissions gouvernementales, espèce particulière de lieu neutre, constituent des espaces où les pensées dominantes sont transformées en vision officielle : s'exprimant dès lors dans des paroles d'institutions officielles, elles bénéficient de la propriété d'universel dont celles-ci sont les dépositaires (Bourdieu, 2012). Ici nous considérerons un travail, commandé par le ministre de la Santé, mené par une mission d'inspecteurs des affaires sociales en vue d'élucider les dessous du scandale du Mediator. 
Reste à caractériser la relation entre moment critique d'une part, et concordance de logiques d'action d'agents appartenant à des champs distincts d'autre part. Par moment critique, ou crise, nous entendons une situation où sont mises en faillite les institutions (règles, procédures et routines) qui assurent la régulation d'un ou de plusieurs champs (Boyer, 2003). Ainsi, une crise peut être "locale ", c'est-à-dire propre à un champ ; elle peut aussi être "générale ", quand elle concerne des champs multiples. Dans ce dernier cas de figure, qui correspond au scandale du Mediator, elle résulte de la coïncidence des effets de plusieurs crises locales (Bourdieu, 1984, p. 211-212). La crise est ainsi le fruit de mobilisations d'agents appartenant à des champs distincts ${ }^{4}$. Une précision s'impose : si les moments critiques se distinguent par l'intensité des conflits, les institutions qui régissent le fonctionnement des champs font l'objet de luttes incessantes. Parce que les champs sont le fruit de conflits entre des pôles d'attraction antagonistes, leurs agents luttent constamment pour la (re)production de leurs institutions, lesquelles, en organisant leurs fonctionnements, scellent les rapports de force qui les opposent. Segment de l'administration de la Santé, l'Agence du médicament en est une illustration exemplaire : depuis le scandale du Mediator, les règles, procédures et routines par lesquelles ses agents et experts organisent la commercialisation des médicaments font l'objet de dénonciations quasi incessantes.

De par son « effet intégrateur » (Bourdieu, 1984, p. 211), la crise générale alimente un phénomène d'alignement des luttes propres à des champs distincts qui, du fait de leur fermeture relative, se déploient d'ordinaire de manière relativement séparée (Bourdieu, 1984 ; Dobry, 1983, 1986). Ainsi, dans cet article, nous observerons que le moment critique que constitue le scandale du Mediator, dont le déclenchement est orchestré depuis le cabinet du ministre de la Santé, suscite la synchronisation des luttes propres aux champs partisan, administratif, scientifique et journalistique. De telles conditions structurales (la synchronisation des champs induite par une crise) favorisent l'avènement d'un phénomène de concordance de logiques d'action de fractions appartenant à des champs distincts. Ceux qui dans leur champ propre entendent améliorer leur position - les challengers, diraient Fligstein et McAdam (2012) - ont profit à tirer de l'accentuation de la crise que connaît un champ. Ainsi, en rapport à la question de la suspension des médicaments à base de pioglitazone, ceux qui souhaitaient améliorer leur position avaient intérêt à tirer de l'apparition d'un nouveau scandale. Cette situation a notamment eu pour effet de légitimer l'intervention du ministre de la Santé : celui qui avec ses proches a déclenché le scandale du Mediator trouve un nouvel enjeu pour se faire valoir.

Sur cette base analytique, trois mécanismes peuvent être distingués pour analyser la décision de suspension des médicaments à base de pioglitazone :

- Le premier porte sur la synchronisation des luttes propres aux différents champs (partisan, administratif, journalistique, scientifique). Ce phénomène est le fruit du déclenchement du scandale du Mediator : orchestrée depuis le cabinet du ministre de la Santé, cette stratégie - qui a pris pour appui une mission de l'Inspection générale des affaires sociales (IGAS) - visait à construire la légitimité ministérielle en condamnant l'Agence du médicament pour la refonder.

4. Ce que Michel Dobry appelle des «mobilisations intersectorielles » $(1983,1986)$. 
- Le deuxième porte sur l'avènement d'un phénomène de concordance de logiques d'action de scientifiques, mais surtout de journalistes de la santé et de parlementaires en faveur de la suspension des médicaments à base de pioglitazone. Ces agents trouvaient un intérêt à accentuer la crise, en construisant cette question comme un élément du scandale du Mediator.

- Enfin, le troisième porte sur les effets du phénomène de concordance : il a déclenché l'intervention du ministre de la Santé qui trouvait une occasion de montrer ce que sera l'agence réformée. Opérant un violent déclassement de l'agence, il facilitait aussi l'imposition de la décision ministérielle à ses experts et agents.

\section{Encadré 1. Sources et méthodes}

Cette enquête repose, premièrement, sur seize entretiens semi-directifs conduits entre juin 2015 et décembre 2015. Ils ont concerné :

- la chargée de mission «médicament » de la sous-direction «Politique des produits de santé et qualité des pratiques et des soins ", direction générale de la Santé, ministère des Affaires sociales et de la Santé ;

- la sous-directrice du département des produits de santé de la CNAM-TS - auparavant conseillère technique pour les produits de santé dans le cabinet de Xavier Bertrand;

- un statisticien du département "Études de santé publique ", direction de la stratégie, des études et de la statistique, CNAM-TS;

- le directeur de l'évaluation médicale de l'AFSSAPS, représentant de la France à la European Medicines Agency (EMA) ;

- le chef de l'unité "Évaluation clinique des médicaments en cancérologie, hématologie, immunologie, dermatologie, orphelins et des médicaments dérivés du sang » de I'AFSSAPS, représentant de la France à l'EMA ;

- un directeur de recherche en épidémiologie de l'Institut national de la santé et de la recherche médicale (INSERM), expert à la Haute Autorité de santé (HAS) - futur directeur de la stratégie et des affaires internationales de l'Agence nationale de sécurité du médicament ;

- le chef de service de l'évaluation médicale de la HAS;

- un médecin des hôpitaux, praticien hospitalier, expert à la HAS ;

- un professeur des universités, praticien hospitalier en pharmacologie, expert à I'AFSSAPS et à la HAS ;

- un professeur des universités, praticien hospitalier en diabétologie;

- un maître de conférences, praticien hospitalier en diabétologie, expert ad hoc à I'AFSSAPS ;

- un journaliste de Libération, un journaliste du Monde ;

- la directrice des affaires pharmaceutiques de Takeda France ;

- la responsable de la communication de Takeda France.

Cette enquête repose, deuxièmement, sur des sources écrites. Leur abondance nous a permis de compléter l'enquête par entretien qui, nombreux étant ceux qui ont refusé de répondre à nos sollicitations pourtant multiples, a été quelque peu limitée. Nous avons consulté les relevés des séances de deux instances-clés de l'AFSSAPS au cours desquelles la question de la suspension des médicaments à base de pioglitazone a 
été discutée (commission nationale de la pharmacovigilance et commission d'autorisation de mise en marché), les rapports parlementaires sur le Mediator ${ }^{\circledR}$ et les relevés des auditions sénatoriales (les relevés des auditions de l'Assemblée nationale n'étant pas accessibles). Notre attention a aussi porté sur la presse généraliste : nous avons dépouillé des articles de presse nationale (dont Le Monde, Le Figaro, Libération) portant sur lesdits médicaments. Nous avons enfin consulté la presse médicale (Prescrire, The Lancet) et des revues académiques (Drug Safety, Diabetes Care, Thérapie).

Notre démonstration empirique s'organise en trois temps. Nous resituons d'abord la définition de la stratégie ministérielle qui, élaborée en réponse aux luttes qui entouraient la gestion administrative du Mediator, a déclenché le scandale. Nous montrons ensuite que, construite comme un élément du scandale du Mediator, la question de la suspension des médicaments à base de pioglitazone a suscité un phénomène de concordance de logiques d'action de scientifiques, mais surtout d'élus et de journalistes en sa faveur. Enfin, nous envisageons la façon dont la décision ministérielle a été imposée aux experts de l'AFSSAPS, mais aussi consolidée vis-à-vis de Takeda et de la Commission européenne. Ce sera l'occasion de montrer que cette décision ne se comprend qu'en rapport aux enjeux propres au champ partisan.

\section{L'avènement d'une stratégie ministérielle}

Quand Xavier Bertrand prend les commandes du ministère de la Santé, le scandale du Mediator couve. De l'observation des conditions structurales dans lesquelles son cabinet déploie son activité stratégique découle deux éléments : le premier est que le choix qui est fait par ses membres de faire advenir le scandale, dont l'avènement leur paraît inévitable ; le second est que la mise en accusation de l'AFSSAPS - des règles, procédures et routines par lesquelles ses agents régulent le marché du médicament - constitue pour eux la seule stratégie possible.

\section{« Le scandale du Mediator »: I’héritage du ministre}

Quand il reprend le 14 novembre 2010 les rênes du ministère de la Santé ${ }^{5}$, Xavier Bertrand occupe une position-clé dans le champ partisan : pour accomplir son nouveau mandat, il abandonne le secrétariat général de l'Union pour un mouvement populaire (UMP), fonction qui d'ordinaire constitue un tremplin pour les prétendants à la présidence de la République. Le nouveau ministre de la Santé n'entend cependant pas jouer le rôle de second couteau. Faire éclater ce qui deviendra le scandale du Mediator lui permet d'engager un coup politique.

Jusqu'à la nomination de Xavier Bertrand, il n'y a pas à proprement parler de scandale. Les luttes qui portent sur l'opportunité d'un retrait du marché du Mediator sont circonscrites au champ scientifique et au champ de l'administration du Médicament. Ainsi, Jean-Louis Montastruc - un professeur de pharmacologie sur lequel nous reviendrons - publie en 2006

5. Rappelons que Xavier Bertrand avait occupé cette fonction de 2005 à 2007. 
avec ses collègues un article précurseur dans lequel ils décrivent un cas de valvulopathie suscité par l'administration de l'antidiabétique (Noize et al., 2006). En 1999, puis en 2006, la commission de la transparence de la Haute Autorité de santé (HAS), instance qui conseille le ministre de la Santé quant à l'opportunité de la prise en charge des médicaments par la Sécurité sociale, se prononce en faveur du déremboursement du Mediator ${ }^{6}$. Les choses changent en 2010. Le 5 juin, Anne Jouan - journaliste de la santé au Figaro, incarnation typique de la figure de l'expert critique décrite par Marchetti (2010) convoitant le leadership de l'équipe médicale du Monde - publie un article ${ }^{7}$ dans lequel elle révèle le combat judiciaire engagé par la firme Servier à l'encontre d'Irène Frachon, pneumologue brestoise, qui publie alors un livre ${ }^{8}$ intitulé Mediator $150 \mathrm{mg}$ : combien de morts ? Le 24 août, Gérard Bapt - député du Parti socialiste (PS) de Haute-Garonne, sur lequel nous reviendrons aussi publie dans Le Monde une tribune dont le titre reprend à peu près le titre du livre d'Irène Frachon, "Mediator : combien de morts ${ }^{9}$ ? " Au même moment, des médecins spécialistes de santé publique de la CNAM-TS mènent une étude pharmaco-épidémiologique sur le Mediator que les dirigeants de l'AFSSAPS ont commandée, dans le cadre d'une réévaluation du rapport entre ses bénéfices et risques. Cette étude est initiée par la toute nouvelle cellule "Plan de gestion des risques pharmaco-épidémiologiques " (PGR-PEPI), mise en place en 2009, notamment avec l'appui de Jean-Louis Montastruc. Quand Xavier Bertrand arrive à la tête du ministère de la Santé, les résultats de cette étude - que les dirigeants de l'AFSSAPS, de la CNAM-TS et de la DGS tentaient tant bien que mal de garder secrète ${ }^{10}-$ sont connus : l'administration du Mediator serait à l'origine de 500 décès par valvulopathie. Xavier Bertrand et ses conseillers - qui ont une bonne connaissance de l'administration de la Santé dont ils furent aux commandes quelques années auparavant - savent le scandale imminent. Dès le lendemain de la nomination du ministre se tient la conférence de presse lors de laquelle les responsables de l'AFSSAPS annonceront les résultats de l'étude qu'ils avaient commandée à la CNAM-TS. L'enchaînement des événements n'est pas un hasard du calendrier. L'AFFSAPS et la CNAM-TS sont placées sous la tutelle du ministre de la Santé : compte tenu de l'enjeu, leurs dirigeants agissent alors en étroite concertation avec son cabinet ${ }^{11}$. Ses membres savent que la dimension tragique de l'annonce va déclencher un scandale ${ }^{12}$ dont la prise en charge sera au cœur du mandat ministériel dont le point d'orgue sera la «loi Bertrand». Votée le 29 décembre 2011, celle-ci (dite loi sur le renforcement de la sécurité sanitaire des médicaments et des produits de santé) abolira l'AFSSAPS pour fonder l'Agence nationale de sécurité sanitaire des médicaments et des produits de santé (ANSM). La disqualification de l'Agence du médicament est en effet au cœur de la stratégie ministérielle qui s'élabore alors.

6. HAS, Avis du 10 mai 2006, Paris ; HAS, Avis du 17 novembre 1999.

7. A. Jouan, "Servier dans la tourmente judiciaire », Le Figaro, 5 juin 2010.

8. I. Frachon, « Mediator $150 \mathrm{mg}$ : combien de morts ? », Brest, Dialogues, 2010.

9. G. Bapt, « Mediator : combien de morts ? ”, Le Monde, 24 août 2010.

10. L'existence de cette étude gardée secrète a été révélée par A. Jouan ("Le Mediator serait responsable de 500 à 1000 morts ", Le Figaro, 13 octobre 2010).

11. Entretien, médecin spécialiste de santé publique, CNAM-TS.

12. Entretien, conseiller technique du ministre. 


\section{Fonder l'action ministérielle en disqualifiant l'AFSSAPS}

Pour le cabinet du ministre, la voie est étroite pour définir une réponse à apporter au scandale qui couve. Du côté du champ bureaucratique, il importe de ne pas discréditer le ministère dont il fut lui-même responsable quelques années auparavant au risque d'entacher sa réputation et celle de ses proches ${ }^{13}$. La chose n'est pas facile : statutairement présent dans les commissions de l'AFSSAPS et de la HAS, le directeur général de la DGS (ou ses suppléants) non seulement participait aux délibérations, mais en plus votait les décisions, parmi lesquelles celles concernant le Mediator. Du côté du champ partisan, il importe de ne pas discréditer les médecins généralistes, dont une part significative prescrivait un médicament pour des fins qui n'étaient pas celles pour lesquelles il avait été commercialisé ${ }^{14}$. En tant que responsable de premier plan de l'UMP, qui plus est ministre de la Santé, Xavier Bertrand - appuyé par ses conseillers - ne veut pas mettre en cause ce qui est constitué par les politistes (e. g. Hassenteufel, 1997) comme une fraction électorale majeure de la droite conservatrice ${ }^{15}$.

Le ministre et ses conseillers font donc le choix de porter la charge contre l'AFSSAPS. Pour cela, le ministre demande le 29 novembre 2011 à l'Inspection générale des affaires sociales (IGAS) une mission d'enquête sur le Mediator. Le choix n'est pas anodin : certes récent ${ }^{16}$, le corps de contrôle des affaires sociales bénéficie d'un prestige en ce qu'il a constitué un tremplin pour la carrière de certains de ses membres qui ont pu faire valoir leurs savoir-faire dans des cabinets ministériels et/ou à la tête de directions d'administration centrale (Hassenteufel et al., 1999). Aquilino Morelle, qui prendra en charge la mission, compte parmi ceux-ci. Ce diplômé de l'Institut d'études politiques de Paris, de la faculté de médecine de Paris et de l'École nationale d'administration jouit d'une grande autorité : conseiller technique de Bernard Kouchner de 1992 à 1993 quand celui-ci était ministre de la Santé, il est d'ordinaire perçu comme un maître d'œuvre - avec Martin Hirsch et Didier Tabuteau - du phénomène d'"agencification" que connut l'administration de la Santé dans les années 1990. Cadre du PS, il deviendra la «plume» du Premier ministre Lionel Jospin, de 1998 à 2002, puis du président François Hollande, de 2012 à 2014. Pour Xavier Bertrand et ses conseillers, jouer la carte "Aquilino Morelle", c'est construire l'apparente neutralité du rapport que la mission produira : d'abord en la parant d'une légitimité experte, celle qui est attachée à une figure de la santé publique appartenant à un corps d'inspection reconnu. Ensuite en la dotant d'une légitimité trans-partisane : par cet appui, l'homme de l'UMP dispose d'une caution du PS de haut rang.

La commande détermine le contenu. Corps de contrôle des affaires sociales, I'IGAS portera par définition son regard sur l'AFSSAPS ; les ministres de la Santé qui furent en poste, comme le corps médical ou Servier, seront largement exclus du périmètre de l'enquête. La critique sera acerbe : s'il doit sa carrière à la santé publique, Aquilino Morelle entretient un rapport ambivalent avec l'administration de la Santé qu'il s'est donné pour

13. Chose qui arrivera peu après. Par exemple : «Deux conseillers de Xavier Bertrand en 2006 avaient des liens avec Servier ", Le Monde, 11 janvier 2011.

14. Le Mediator, un antidiabétique, était principalement prescrit pour ses vertus "anorexigènes ", soit pour gagner à soi une taille de guêpe.

15. Entretien, conseiller technique du ministre.

16. Le corps fut créé en 1967. 
mission de réformer. II est, avec Didier Tabuteau, à l'origine d'une doctrine réformatrice dont le point de départ est la faiblesse de ce segment administratif (Tabuteau, 1996, 1994 ; Morelle, 1996). La raison en est que celui-ci ne dispose pas de grands corps techniques: ses effectifs sont peu nombreux, son expertise propre peu développée et son prestige faible. L'administration de la Santé occupe pour eux une position dominée, tant vis-à-vis d'autres segments administratifs que de ses interlocuteurs. En identifiant les "défaillances " de l'administration de la Santé en général, et de l'AFSSAPS en particulier, la mission d'enquête de l'IGAS va actualiser ce tableau. Le 15 janvier 2011 - jour où, officiellement, le rapport de I'IGAS est rendu - Xavier Bertrand donne une conférence de presse $^{17}$. Après avoir souligné " la qualité du travail et la quantité du travail accompli par la mission " à l'origine d'un rapport jugé rigoureux, le ministre condamne - outre Servier l'administration de la Santé : «le rapport montre clairement que notre police du médicament a failli à sa mission » (p. 1). Dans le rapport d'Aquilino Morelle et de ses collègues, l'AFSSAPS, « inexplicablement tolérante à l'égard d'un médicament sans efficacité réelle ", est en effet le cœur de la critique. L'agence est dépeinte comme une "bureaucratie sanitaire " : " une structure lourde, lente, peu réactive, figée » (p. 13). Dépourvue d'expertise propre, reposant sur des scientifiques entretenant des liens étroits avec les firmes pharmaceutiques, elle est "structurellement et culturellement dans une situation de conflit d'intérêts» (p. 15). Fort de ce rapport, Xavier Bertrand annonce lors de la conférence de presse sa volonté de "rebâtir un nouveau système du médicament " par des «mesures radicales " (p. 1). Dans le moyen terme, " dès cette année » (p. 6), il déclare vouloir rebâtir l'agence (« il y a une évidence : nous ne pouvons conserver l'AFSSAPS en l'état »- p. 3). Dans le court terme, le ministre déclare vouloir s'impliquer dans la régulation du marché du médicament. Celui-ci proclame s'engager à faire le "ménage dans la pharmacopée ", jugeant «qu'en France, il y a trop de médicaments ${ }^{18}$ ". II demande aux responsables de l'AFSSAPS de dresser un bilan des médicaments à risque qui publieront quelques semaines plus tard une liste de " 77 médicaments sous surveillance ».

Par la stratégie qu'ils déploient, le ministre et ses conseillers vont réaliser un véritable tour de force dont les effets porteront notamment dans les champs partisan, bureaucratique et journalistique: ils prennent la main sur la temporalité du scandale qu'ils font éclater ; ils prennent aussi la main sur son cadrage, qui représente le scandale comme le fruit d'une agence défaillante. Ainsi, les missions parlementaires sur le Mediator, qui seront mises en place quelques jours après la conférence de presse du ministre (celle de l'Assemblée nationale débutera le 26 janvier, celle du Sénat le 31), consacreront les grandes lignes du rapport de I'IGAS. En fondant son autorité dans le champ partisan comme bureaucratique en disqualifiant l'AFSSAPS, le ministre fait donc le choix de contribuer de manière décisive à sa mise en crise. Plus qu'un « fusible ${ }^{19}$ ", l'agence est le " détonateur » de l'action ministérielle.

17. Xavier Bertrand, conférence de presse sur le Mediator, 15 janvier 2011.

18. Pierre Bienvault, "Le Gouvernement fait le ménage dans les armoires à pharmacie ", La Croix, 23 juin 2011.

19. Pour reprendre l'expression de Buton, Pierru (2012, p. 68). 


\section{Les médicaments à base de pioglitazone comme élément du scandale}

Au même moment, un autre scandale couve. Portée au sein de l'AFSSAPS par JeanLouis Montastruc, la question de la suspension des médicaments à base de pioglitazone fait l'objet de l'opposition de ses pairs, décision qui reflète le rapport de force traditionnel au sein de l'agence, au-delà du sein du champ scientifique. Faisant le choix de contourner l'opposition de l'agence, ce pharmacologue mobilise un système d'alliances très proche de celui qui a fait émerger le scandale du Mediator, en suscitant un nouveau. Initié par les mêmes agents, celui-ci est présenté comme une « réplique " de l'affaire du Mediator. Une concordance de logiques d'action d'élus et de journalistes en faveur de la suspension des médicaments à base de pioglitazone émerge sur cette base.

\section{La mobilisation contre les médicaments à base de pioglitazone : l'échec de la voie procédurale}

La décision de suspension des médicaments à base de pioglitazone plonge ses racines dans une mobilisation que conduit Jean-Louis Montastruc, un scientifique de haut rang. Professeur de pharmacologie, chef du service de pharmacologie médicale et clinique du centre hospitalier universitaire $(\mathrm{CHU})$ de Toulouse et responsable du centre régional de pharmacovigilance (CRPV) de Midi-Pyrénées, celui-ci connaît une carrière brillante. Fils de Paul Montastruc, d'ordinaire considéré comme "un des pères fondateurs de la pharmacologie française ${ }^{20}$ ", Jean-Louis est depuis 2007 membre correspondant de l'Académie de médecine à laquelle il sera élu en 2013. Partisan d'une "pharmacologie globale ${ }^{21}$ ", son travail vise à dépasser les clivages disciplinaires pour évaluer les risques et les bénéfices des médicaments à l'aune des méthodologies clinique et épidémiologique. Celui qu'un de ses collègues qualifiait moqueusement d'«ange blanc de la pharmacologie ${ }^{22}$ " prône aussi une recherche "indépendante " de l'industrie pharmaceutique, donc à l'encontre des pratiques dominantes de ses pairs, une posture qu'exprime sa collaboration ancienne à la revue médicale Prescrire ${ }^{23}$.

\section{Encadré 2. La pharmacologie : la science du médicament}

La pharmacologie, la science du médicament (Dangoumau, 2006), se développe en France dans les années 1960 quand l'industrie pharmaceutique prend son essor. Elle emprunte à différentes disciplines scientifiques (pharmacie, chimie, biologie, génétique, thérapeutique) et comprend différentes spécialités pour investir les mécanismes d'interactions entre substances actives et organismes. Parmi celles-ci figure la pharmacologie clinique, dont les tenants dominent la discipline jusque dans les années 1990. Ce savoir porte sur l'évaluation des effets du médicament chez l'homme

20. Selon Bernard Bégaud, professeur de pharmacologie à l'université de Bordeaux. B. Bégaud, "Hommage à monsieur le professeur Montastruc ", Thérapie, 62 (3), 2007, p. 275.

21. Entretien, professeur de pharmacologie.

22. Entretien, professeur de pharmacologie.

23. Journal qui se targue d'être la seule revue professionnelle indépendante des crédits des firmes. 
lors d'essais cliniques conduits en vue de l'obtention d'une autorisation de mise en marché : il s'agit donc d'apprécier l'efficacité intrinsèque d'un médicament dans des conditions qui visent à minimiser les facteurs de variation (associations médicamenteuses, âges, etc.). Apparue dans les années 1990, la pharmaco-épidémiologie acquiert pour sa part ses lettres de noblesse académiques au début des années $2000^{24}$. Elle vise l'évaluation en conditions réelles de l'efficacité, de l'usage et de la tolérance des médicaments mis sur le marché (Jones, Kingery, 2014 ; Bégaud, Dangoumau, 2000). Aux essais cliniques sont préférées les études observationnelles, tournées vers de grandes populations, sur lesquelles reposent des analyses statistiques.

Depuis quelques années, Jean-Louis Montastruc mène des recherches sur la pioglitazone en partenariat avec Dominique Hillaire-Buys, médecin au CHU de Montpellier et responsable du CRPV de Languedoc-Roussillon. Repérant quelques biais dans une étude financée par Takeda dans le cadre de la procédure européenne de mise en marché de ses spécialités à base de pioglitazone, les deux pharmacologues établissent une corrélation entre leur usage et la survenue de cancers de la vessie. Leurs résultats sont publiés en 2010 ; d'autres publications suivront. En septembre 2010, une alerte de la prestigieuse Food and Drug Agency (FDA) conforte leurs résultats, ses experts estimant que l'administration des médicaments à base de pioglitazone favorise un risque accru de cancers de la vessie ${ }^{25}$. N'oublions pas qu'au même moment, les résultats de l'étude sur le Mediator conduite par la CNAM-TS à la demande de la cellule PGR-PEPI dont Jean-Louis Montastruc est membre se dessinent. En plus du terrain pharmacologique, celui-ci décide donc d'investir avec sa collègue Dominique Hillaire-Buys un nouvel espace de luttes : c'est à travers deux instances de l'AFSSAPS dans lesquelles ils siègent qu'ils dénoncent les risques que suscite selon eux l'usage des médicaments à base de pioglitazone : le Comité technique de la pharmacovigilance (CTPV), qui assure le contact avec les CRPV ; la cellule « Plan de gestion des risques pharmaco-épidémiologiques » (PGR-PEPI). Les événements se déploient dès lors quand le scandale du Mediator a été déclenché. En novembre 2010, Dominique Hillaire-Buys alerte les membres de la CTPV qui estiment que les données qu'elle présente justifient une saisie de la cellule PGR-PEPI. En janvier 2011, ses membres - dont Jean-Louis Montastruc conseillent le directeur de l'agence, Jean Marimbert, de mettre en place une étude pharmaco-épidémiologique que conduira la CNAM-TS, à l'image de celle conduite sur le Mediator. Jean Marimbert suit le conseil de la cellule PGR-PEPI. La procédure mérite précisions : créée en 2009, la cellule PGR-PEPI vise l'introduction de la pharmaco-épidémiologie dans l'arsenal méthodologique de l'AFSSAPS que dominait l'approche clinique ${ }^{26}$. Au même moment, le directeur de l'AFSSAPS fait aussi le choix de suivre la procédure européenne, en alertant l'agence irlandaise, laquelle - au sein de la European Medicines Agency (EMA) est en charge de délivrer les autorisations de mise en marché des médicaments à base de

24. Entretien, professeur de pharmacologie.

25. FDA, Drug Safety Communication: Ongoing Safety Review of Actos (Pioglitazone) and Potential Increased Risk of Bladder Cancer after Two Years Exposure, 2010. La découverte des deux pharmacologues français n'était pas isolée : au même moment, une étude que finançait Takeda dans le cadre de la procédure américaine de mise en marché de ses spécialités à base de pioglitazone permet à des chercheurs de tirer des conclusions similaires (Lewis et al., 2011).

26. Entretiens, AFSSAPS. Voir aussi Sommet et al. (2007). 
pioglitazone ${ }^{27}$. En mars, la décision est prise de réévaluer lesdites spécialités, en intégrant les données scientifiques nouvelles qui ont été versées au dossier ; les résultats de ce travail sont attendus pour juin.

Aucune des procédures ne contente les membres de la Commission nationale de la pharmacovigilance (CNPV) qui se réunit à la fin du mois de mars : Dominique Hillaire-Buys présente à ses membres ses inquiétudes, désormais aggravées par la recension de 15 notifications spontanées de cas de cancers de la vessie contractés par des patients consommant des médicaments à base de pioglitazone. La pharmacologue emporte la conviction de ses pairs : selon eux, des "mesures immédiates ${ }^{28}$ " s'imposent - à savoir la suspension desdits médicaments. Ils alertent donc leurs collègues de la commission d'AMM qui inscrivent le dossier à l'agenda de leur séance d'avril. Ces derniers, après avoir écouté Dominique Hillaire-Buys, s'opposent cependant très largement à la suspension des médicaments à base de pioglitazone par 21 voix contre 4 (dont 0 abstention). Ses membres - notamment les agents de l'AFSSAPS en charge du dossier - invoquent la procédure qui fut initiée à l'échelle européenne ${ }^{29}$. Selon eux, si elle est jugée pertinente, l'étude demandée à la CNAM-TS pourra être versée au dossier. À l'argument procédural s'en ajoute un autre, scientifique : cliniciens pour la majoritée ${ }^{30}$, les experts de la commission d'AMM regardent avec scepticisme (sinon dédain) les données scientifiques que présente Dominique HillaireBuys, qui relèvent de la pharmacovigilance et de la pharmacologie-épidémiologie ${ }^{31}$. Cette opposition n'est que l'expression du rapport de forces traditionnel qui oppose au sein de l'AFSSAPS la CNPV et la commission d'AMM, rapport de force qui n'est que le reflet de la position subordonnée de la pharmacologie dans le champ médical (Ansaloni et al., 2017). Face à l'opposition de ses pairs, Jean-Louis Montastruc décide d'entrer en dissidence.

\section{La stratégie du contournement : une concordance de logiques d'action contre les médicaments à base de pioglitazone}

La démarche n'est guère surprenante de la part du pharmacologue toulousain : s'il a occupé des positions tant à l'AFSSAPS qu'à la HAS, celui-ci n'hésite pas à prendre la parole dans la presse nationale pour critiquer haut et fort les règles et procédures qui guident le travail de leurs experts. Ainsi, à la fin de l'été 2010, un article du Figaro titrait, sans nuance : "Montastruc : "il faut revoir l'évaluation des médicaments"32 ". Cette fois-ci, il prend sa plume pour alerter des députés du PS de Haute-Garonne, alors dans l'opposition, avec lesquels il

27. Instance de l'EMA chargée de la préparation des avis sur les médicaments humains, le Committee for Medicinal Products for Human Use (CHMP) se compose à part égale de représentants des agences nationales : chaque procédure de mise en marché est instruite par deux représentants nationaux dont les avis sont discutés en plénière.

28. AFSSAPS, Commission nationale de pharmacovigilance, 29 mars 2011, $15 \mathrm{p}$.

29. AFSSAPS, Commission d'autorisation de mise en marché, 7 avril 2011, 35 p.

30. II en est ainsi de Philippe Lechat - professeur de pharmacologie, médecin cardiologue à l'hôpital de la Pitié-Salpêtrière - alors aux commandes de la Direction de l'évaluation des médicaments de l'AFSSAPS.

31. Selon un agent de l'AFSSAPS: "Beaucoup ne croient qu'aux essais cliniques. S'ils n'ont pas ça, ils ne croient pas. Seule compte l'expérimentation, ils ne tiennent pas compte de l'observation. Mais bien souvent vous ne pouvez pas expérimenter. II faut donc se contenter d'observer. C'est une école de pensée " (entretien).

32. Martine Perez, "Montastruc : “il faut revoir l'évaluation des médicaments” ", Le Figaro, 29 septembre 2010, p. 12. 
entretient une certaine proximité : Gérard Bapt - médecin cardiologue ; Catherine Lemorton - pharmacienne, présidente de la commission des affaires sociales. Devenu président de la commission d'information de l'Assemblée nationale sur le Mediator, Gérard Bapt réagit au quart de tour. Celui qui convoite le poste de ministre de la Santé lors d'une prochaine alternance, espérant donc dans l'immédiat valoriser sa position dans le champ partisan, est désormais un protagoniste du procès politique qui est en train de se tenir.

Par une dépêche à l'Agence France-Presse du 19 avril, Gérard Bapt porte la question de la suspension des médicaments à base de pioglitazone à la connaissance des journalistes. Son diagnostic est sans ambiguiité : " vous remplacez le mot Mediator par le mot Actos et vous avez les mêmes interlocuteurs qui vous disent les mêmes choses $^{33}$ ". Selon le député de HauteGaronne, fustigeant la commission d'AMM de l'AFSSAPS qui, quelques jours plus tôt, s'est prononcée contre la suspension des médicaments à base de pioglitazone, il ne s'agit ni plus ni moins d'un «remake du Mediator ». Quelques jours plus tard, les journalistes des principaux organes de presse nationale se saisissent de la question. Autre protagoniste du scandale du Mediator, Anne Jouan - journaliste au Figaro - soulève le lièvre. Par un article publié le 27 avril, elle relaie le diagnostic de Gérard Bapt : " on prend les mêmes et on recommence. Ou comment, alors que le scandale du Mediator a éclaté voilà bientôt un an, l'histoire semble se répéter ${ }^{34}$ ". La journaliste dénonce l'immobilisme des membres de la commission d'AMM qui ont voté contre la suspension de l'usage des médicaments à base de pioglitazone : « il est intéressant de relever que ceux qui s'opposent à la suspension immédiate d'Actos et Competact sont les mêmes qui freinaient pour le retrait du Mediator ». Dans la foulée, le 28 avril, des journalistes de presse et de télévision se saisissent de la question, suggérant un même parallèle ${ }^{35}$.

Gérard Bapt atteint sa cible : les députés et sénateurs qui mènent les auditions parlementaires sur la gestion du Mediator se saisissent de la question de la suspension des médicaments à base de pioglitazone, laquelle n'avait pas pénétré les instances parlementaires ${ }^{36}$. Pour ceux qui sont à l'origine de ce nouveau scandale, comme pour les députés et sénateurs qui le relaient, la question de la suspension des médicaments à base de pioglitazone n'est autre qu'une réplique du scandale du Mediator. Quelle que soit leur affiliation politique, les médecins qui dans les commissions parlementaires monopolisent les prises de parole sur la question du Mediator sont unanimes pour condamner l'administration de la Santé, I'AFSSAPS en particulier, pour sa gestion de l'antidiabétique ${ }^{37}$. La collusion entre

33. Agence France-Presse, «Deux médicaments antidiabétiques, l'Actos et le Competact, pourraient voir leur vente suspendue ", 19 avril 2011.

34. Anne Jouan, "Deux antidiabétiques créent une nouvelle polémique », Le Figaro, 27 avril 2004.

35. Benoît Le Corre, "Actos et Competact, les nouveaux Mediator ? ", Le Parisien, 28 avril 2011; Camille Caldini, "Après le Mediator, deux autres antidiabétiques suspectés ", L'Express, 28 avril 2011 ; " Gérard Bapt s'insurge contre Actos et Compectact : vers les nouveaux Mediator? ", Mediapart, 28 avril 2011 ; " Deux médicaments antidiabétiques, l'Actos et Competact, pourraient voir leur vente suspendue ", France 2, 28 avril 2011.

36. Outre les interventions de Jean-Louis Montastruc lors de ses auditions à l'Assemblée nationale et au Sénat, les $1^{\text {er }}$ et 2 mars, voir : Assemblée nationale, Rapport d'information. Le Mediator et la pharmacovigilance, Paris, 2011, p. 70.

37. Les parlementaires font bloc, comme le relève Gérard Bapt dans l'avant-propos du rapport remis par l'Assemblée nationale, saluant la capacité des membres de la commission qu'il dirigea à "surmonter les clivages partisans " (Assemblée nationale, Rapport d'information. Le Mediator et la pharmacovigilance, Paris, 2011, p. 7-8). 
l'industrie et ses experts sur les avis desquels reposait l'autorisation de mise en marché du Mediator est pour ces parlementaires au fondement d'une gestion administrative qui a coûté la vie à des centaines de patients. La question de la suspension des médicaments à base de pioglitazone trouve une même réponse : la présence sur le marché de cette molécule dangereuse n'est autre que le fait d'une agence dont les experts, pétris de "conflits d'intérêt ", sont à la botte des firmes. Le diagnostic débouche sur un appel à une autorité supérieure, celle du ministre de la Santé. Ainsi, le 6 juin, lors de son audition, le président de la commission d'enquête sénatoriale interpelle Xavier Bertrand, le sommant de prendre en main la question de la suspension des médicaments à base de pioglitazone :

«Prenons le cas de l'Actos, qui ressemble à bien des égards au Mediator [...]. II s'agit peut-être d'un futur Mediator. Vous devriez avoir la possibilité de remettre en cause la décision de maintien de l'Actos sur le marché prise par le directeur général de l'AFSSAPS. Peut-être conviendrait-il d'octroyer davantage de pouvoir au ministre. Si une affaire similaire à celle du Mediator apparaît concernant l'Actos, ce sera, en effet, certainement le ministre qui sera davantage responsable que le directeur général de l'AFSSAPS [...]. L'Actos représente la parfaite illustration de la situation dans laquelle nous nous trouvons. Elle répète le Mediator. Sur ce plan, nous n'avons pas progressés ${ }^{38}$.

Ainsi, faisant le choix de contourner l'opposition de ses pairs qui siégeaient dans la commission d'AMM, Jean-Louis Montastruc a mobilisé un système d'alliances identique à celui qui fut à l'origine du scandale du Mediator. La question de la suspension des médicaments à base de pioglitazone est alors devenue un élément dudit scandale, sa réplique précisément. Le diagnostic a reçu la validation des journalistes comme des députés et sénateurs qui conduisaient à ce moment le procès du Mediator. Une concordance de logiques d'action entre des agents appartenant à des fractions de champs multiples a donc fait jour en faveur de la suspension des médicaments à base de pioglitazone.

\section{Une décision ministérielle tournée vers le champ partisan}

La mise en demeure du ministre est quelque peu artificielle : après la remise du rapport de l'IGAS, celui-ci s'était engagé à faire le " ménage dans la pharmacopée française ". La question de la suspension des médicaments à base de pioglitazone est l'occasion de traduire dans les institutions du champ bureaucratique cet engagement proclamé. Si, une fois appréciées les conditions de possibilité de la décision de suspension, l'imposition de la décision ministérielle à une agence qui fait l'objet d'un violent déclassement est aisée, reste au ministre et à ses conseillers à asseoir leur décision, tant vis-à-vis des autorités européennes que de Takeda. L'observation de l'action ministérielle révèle combien celle-ci ne se comprend qu'au regard des enjeux propres au champ partisan.

38. [www.senat.fr/compte-rendu-commissions/20110606/mediator.html\#toc6]. 


\section{La mise au pas de l'agence}

Dès que la question de la suspension des médicaments à base de pioglitazone est inscrite sur les agendas partisan et médiatique, le cabinet du ministre examine les conditions de possibilité sanitaire d'une telle décision. Un cercle d'expertise ad hoc est établi. À son cœur figure Christelle Ratignier-Carbonneuil : médecin spécialiste en toxicologie, chargée de recherche à l'Institut national de la santé et de la recherche médicale (INSERM), elle fut en poste trois ans à l'AFSSAPS en tant que chef de l'évaluation clinique au sein de la Direction de l'évaluation des médicaments. Cette conseillère travaille en relation directe avec Dominique Maraninchi, le nouveau directeur de l'agence que Xavier Bertrand a nommé le 22 février. Ils s'attachent à lever une double interrogation ${ }^{39}$ : la première est celle de savoir si la décision de suspension des médicaments à base de pioglitazone est susceptible de faire du tort aux quelque 230000 patients alors sous traitement. Dominique Maraninchi consulte quelques diabétologues. Parmi eux, un accord existait pour dire que lesdits médicaments n'étaient guère indispensables: si leur efficacité thérapeutique est contestée, la classe des antidiabétiques se caractérise (selon le jargon médical) par un nombre d'innovations élevé (Pichetti et al., 2013) ; les alternatives ne manquent pas. La seconde question porte sur la pertinence des données scientifiques qui établissent une corrélation entre médicaments à base de pioglitazone et survenue de cancers de la vessie. Notamment les conseillers du ministre s'interrogent sur la qualité de l'étude menée par la CNAM-TS. Avant sa publication, Dominique Maraninchi consulte un de ses proches, Mahmoud Zureik - épidémiologiste, directeur de recherches à l'INSERM : celui qui a participé quelques mois plus tôt à l'étude sur le Mediator que l'AFSSAPS avait demandée à la CNAM-TS juge le nouveau travail de bonne facture. Dès lors, le ministre décide d'imposer la suspension des médicaments à base de pioglitazone aux membres de la commission d'AMM.

Contraindre les experts de la commission d'AMM sera chose aisée. L'AFSSAPS, qui a été condamnée à mort par le ministre quelques mois plus tôt, fait l'objet d'un violent déclassement. Les agents de l'agence comme ses experts sont sur la sellette : lors des auditions parlementaires, dans les colonnes de la presse, ils sont mis au banc des accusés pour leur complaisance supposée à l'égard de Servier qui aurait occasionné plusieurs centaines de morts. Quand il entre en fonction, le nouveau directeur de l'AFSSAPS limoge quelques responsables, notamment celle qui était chef de la surveillance du risque, du bon usage et de l'information sur les médicaments. Dans quelques semaines, les mises en examen seront ouvertes. Quand le directeur de l'AFSSAPS inscrit à l'ordre du jour de la commission d'AMM la question de la suspension des médicaments à base de pioglitazone, les jeux sont faits. Lors de son audition au sénat, le 6 juin - soit trois jours avant la séance de la commission d'AMM, le ministre avait fait savoir quelle serait sa position : si les résultats de l'étude menée par la CNAM-TS penchent en faveur de la suspension, il fera son possible pour acter cette décision :

«Si les études de l'assurance maladie laissent entrevoir le moindre doute, je n'attendrai pas la décision de l'Europe et prendrai mes responsabilités. J'ai évoqué la question avec Dominique Maraninchi qui a adopté la même position [...]. L'Actos ${ }^{\circledR}$ fait partie des médicaments pour lesquels les responsabilités seront prises, sans qu'il soit nécessaire d'attendre un drame dans quelques années. "

39. Entretien, conseiller technique du ministre. 
La contrainte qui pèse sur les experts de la commission d'AMM est d'autant plus grande que le 8 juin, la veille du jour où les membres de la commission d'AMM examinent à nouveau la question de la suspension des médicaments à base de pioglitazone, les conseillers du ministre organisent la fuite du rapport de la CNAM-TS dont les résultats seront présentés le lendemain aux membres de la commission. Le tir atteint sa cible: le jour où se tient la commission, les principaux journaux nationaux couvrent l'événement ${ }^{40}$.

Après présentation des résultats du rapport de la CNAM-TS, qui ne venaient que confirmer les données scientifiques existantes, les membres de la commission d'AMM qui, quelques mois plus tôt s'étaient largement opposés à la décision de suspension, se prononcent en faveur à l'unanimité (25 voix pour, une abstention) ${ }^{41}$. Si quelques pressions ont été exercées sur des membres de la commission d' $\mathrm{AMM}^{42}$, la position structurale de l'agence suffit à ce que le ministre impose à ses membres sa décision. En témoigne un agent du ministère de la Santé :

" ll y a tout un tas de gens qui ne se sont pas exprimés quand il s'agissait de défendre le médicament de crainte d'être accusé d'être pro-labo. C'était clair et net. Alors que la commission d'avant qui datait d'avril, là il y a revirement complet [...]. Tout le monde avait la trouille d'être accusé d'être lié à l'industrie, vendu à l'industrie [...]. À partir du moment où Bertrand s'était exprimé, c'était fini, la messe était dite ${ }^{43}$."

Reste au ministre et à ses conseillers à asseoir une décision de suspension qui, dictée par les logiques partisanes nationales, est juridiquement contestable.

\section{Asseoir une décision fragile}

En effet, les résultats de la réévaluation du rapport entre les bénéfices et les risques que mènent les experts de l'agence européenne du Médicament à la demande de l'AFSSAPS ne sont pas encore connus. S'ils plaident en faveur du maintien sur le marché, la Commission européenne entérinera leur avis, invalidant la décision de l'AFSSAPS qui, de jure, devra autoriser à nouveau la commercialisation des médicaments à base de pioglitazone. Ce sera chose faite quand, le 21 juillet, les experts de l'EMA plaident très largement en faveur de leur maintien sur le marché, un avis que suivra la Commission européenne par une décision du 22 décembre.

Cependant les effets "français" d'un tel camouflet avaient été anticipés. Mahmoud Zureik - un proche de Dominique Maraninchi qui - quand l'Agence nationale de sécurité du médicament et des produits de santé (ANSM) succédera à l'AFSSAPS fera son entrée dans l'agence pour le seconder - avait pris le soin de porter à l'agenda de la commission de la

40. Par exemple: Anne Jouan, "Les antidiabétiques Actos et Competact retirés du marché ", Le Figaro, 9 juin 2011 ; Paul Benkimoun, "Comment l'affaire du Mediator a bousculé la pharmacovigilance française", Le Monde, 9 juin 2011.

41. AFSSAPS, Commission d'autorisation de mise en marché, 9 juin 2011.

42. Le ministre et ses conseillers ont pris soin d'exercer une contrainte directe sur certains cadres de l'agence. L'un d'eux, se remémorant une réunion avec les conseillers du ministre, nous disait : " Je prends l'ascenseur. En bas, je tombe sur Bertrand qui me dit bonjour. II me dit d'emblée: "Je n'attendrai pas la décision de l'Europe, il n'en est pas question." "

43. Entretien, direction générale de la Santé, ministère de la Santé. 
transparence de la Haute Autorité de santé (HAS) la question du déremboursement des médicaments à base de pioglitazone. En tant que membre de la commission, il initie (selon le jargon administratif) une réévaluation de leur service médical rendu, qui sera jugé insuffisant, compte tenu des risques de survenue de cancers de la vessie que lesdits médicaments susciteraient. La commission recommande donc au ministre de la Santé de prononcer le déremboursement des médicaments à base de pioglitazone, le 20 juillet ${ }^{44}$. Ce faisant, ses membres entendent consolider la décision de l'AFSSAPS qu'ils savaient juridiquement faible ${ }^{45}$ : rayer les spécialités à base de pioglitazone de la liste des médicaments pris en charge par la Sécurité sociale, c'est en effet s'assurer qu'elles ne seront plus prescrites. Le ministre de la Santé actera le déremboursement le 22 décembre. Mais, pour les experts de la HAS, il s'agissait aussi de prévenir une éventuelle accusation de complaisance vis-à-vis de l'industrie pharmaceutique ${ }^{46}$. L'arme du déremboursement, brandie par le ministre dans la presse ${ }^{47}$, lui permet d'anticiper toute éventuelle attaque de la part de Takeda comme de la Commission européenne. À quoi bon engager un bras de fer juridique avec l'État français pour casser la décision de suspension des médicaments à base pioglitazone quand ceux-ci, rayés de la liste des médicaments pris en charge par la sécurité sociale, ne seront plus prescrits ? Le calcul se révèle gagnant : ni la Commission européenne, ni Takeda ne donneront de suite judiciaire. Pour la première, ses dirigeants n'ont pas l'habitude de procéder de la sorte ${ }^{48}$; pour la seconde, ses responsables n'entendent pas se mettre à dos l'administration française pour des médicaments dont les brevets arrivent à expiration, signant la chute de leur rentabilité ${ }^{49}$.

$\mathrm{Si}$, à l'échelle nationale, le ministre et ses conseillers ont pris le soin d'asseoir la décision de suspension, ils n'ont cependant pas cherché à faire valoir son bien-fondé auprès des experts de l'agence européenne du Médicament. Alors que d'ordinaire, les États via leurs agences respectives luttent pour améliorer leur position en son sein, en l'espèce, bien peu d'efforts ont été consentis en ce $\operatorname{sens}^{50}$ : si, dans le cadre de la procédure européenne de réévaluation du rapport entre les risques et les bénéfices des médicaments à base de pioglitazone, les experts de la CNAM-TS ont été chargés de présenter les résultats de leur rapport, ceux-ci - maîtrisant mal la langue anglaise, la langue de travail européenne - ont été incapables de répondre en séance aux questions des experts des autres pays qui, au final, ont repoussé leurs arguments. Ainsi, plutôt que de prendre en charge le dossier, le

44. HAS-Commission de la transparence, Avis, 20 juillet 2011.

45. Stratégie classique menée par les autorités sanitaires françaises pour contourner les décisions de mise en marché européennes. Ainsi, Hauray (2005) détaille la façon dont le ministre Bernard Kouchner et son équipe, s'opposant en vain à la mise en marché du Viagra, ont mobilisé l'arme du « déremboursement " pour contrer son développement.

46. Entretien, HAS.

47. Ainsi avait-il déclaré au Figaro: "La France ne veut plus de ces médicaments. Ces deux produits n’ont plus leur place sur le marché français. Si Takeda le reconnaît, c'est mieux, sinon j'évaluerai le risque d'un contentieux avec eux. J'ai aussi en tête la question du déremboursement " (Anne Jouan, "L'Actos menacé de déremboursement ", Le Figaro, 13 novembre 2011).

48. Les agents de la Commission européenne tendent à laisser les firmes qui s'estiment lésées initier les contentieux (Smith, 2013). Le marché pharmaceutique illustre ce positionnement : jusqu'alors, quand des conflits entre les agences européenne et nationale(s) ont surgi, les entreprises - non pas les autorités européennes ont saisi des juridictions nationales et/ou européennes (Debarge, 2012).

49. Entretien, Takeda, Paris.

50. Entretien, CNAM-TS. 
représentant de l'AFSSAPS à l'agence européenne du Médicament a fait le choix de saborder ce qui constituait la principale justification scientifique de la décision de suspension desdits médicaments. Le contraste est grand entre cette réunion, négligée, et la mise en scène de la séance de la commission d'AMM pendant laquelle ses experts ont voté la décision de suspension. Filmée, diffusée sur le site internet de l'agence, présidée par son directeur général (fait exceptionnel présenté comme fait routinier), cette séance visait à représenter ce que serait l'agence réformée : elle donne à voir une agence dont les experts - informés par une expertise publique commandée par l'agence (autre fait exceptionnel présenté comme fait routinier) - débattent en toute "indépendance " (point souligné à de multiples reprises par le directeur de l'agence) des rapports entre les bénéfices et les risques des médicaments à base de pioglitazone, plaçant au premier rang de leurs préoccupations la sécurité sanitaire des patients. Ainsi, pour ceux qui l'ont imposée aux agents et aux experts de la commission d'AMM, cette décision - largement indépendante des luttes bureaucratiques européennes - n'avait de sens qu'en rapport aux logiques partisanes nationales.

\section{Conclusion}

Loin d'être un cas isolé et particulier, la décision de retirer du marché les médicaments à base de pioglitazone éclaire le rapport singulier entre l'État et le champ partisan en France dans ce secteur. Loin d'être « indépendante » du ministre de la Santé, au-delà des luttes partisanes, l'Agence du médicament est étroitement dépendante de l'état de celles-ci, mais aussi de celles qui se déploient au sein des champs bureaucratique, scientifique et journalistique. Leurs évolutions - qui ont favorisé des stratégies d'agents occupant des positions homologues évoluant à leurs interstices - ont favorisé l'avènement du scandale du Mediator sur lequel la question de la suspension des médicaments à base de pioglitazone a été greffée. En a découlé une réforme profonde de l'agence, initiée depuis le cabinet du ministre de la Santé. Deux enseignements font jour : d'abord, l'« expropriation du politique ${ }^{51}$ » que visaient les théoriciens et praticiens de l'" agencification » n'est pas, de toute évidence, advenue, tant s'en faut. Ce résultat mériterait d'être approfondi : il s'agirait notamment d'analyser l'évolution historique du rapport entre l'Agence du médicament (ainsi que l'entité administrative qui l'a précédée, la DPHM) et le champ partisan. Ensuite, se pose la question de la place actuelle qu'occupe le champ économique dans le processus décisionnel de l'agence : si tout indique que les firmes - de par les contrats de recherche qu'elles nouaient avec les spécialistes médicaux et scientifiques qui revêtaient le rôle d'experts - occupaient une place centrale, la « loi Bertrand " semble avoir rebattu les cartes. En durcissant les dispositions sur les conflits d'intérêts, celle-ci a rejeté en dehors de l'agence ceux qui sont à l'avant-garde de la recherche biomédicale ${ }^{52}$. Beaucoup - y compris les partisans d'une «expertise indépendante» - perçoivent ce

51. L'expression est reprise de Max Weber par Didier Tabuteau (2010, p. 37) : membre du cabinet de Bernard Kouchner au sein duquel il participa au mouvement d'" agencification » de l'administration de la Santé, il prit à sa création en 1993 la direction de l'Agence du médicament. II est aujourd'hui responsable de la chaire "Santé " de Sciences Po.

52. À l'échelle d'un pays comme la France, il y a par spécialités pharmaceutiques (médicaments contre le diabète, par exemple) qu'une poignée de scientifiques impliqués dans les débats académiques internationaux. 
mouvement comme le signe d'un appauvrissement de l'expertise sur la base de laquelle sont assises les décisions ${ }^{53}$. En ce sens, rien n'indique que l'éloignement des firmes favorise le renforcement de l'agence. Aux termes de nos développements, une double dépendance structurale ${ }^{54}$ de cette dernière fait jour: en période de crise prévaudrait une dépendance de l'agence aux acteurs qui dominent les champs partisan et journalistique ; en période routinière prévaudrait une dépendance de cette dernière aux agents dominants du champ économique, notamment par le biais des experts et les formes d'expertise qu'elle mobilise au cours de son travail quotidien.

Du point de vue de la théorie sociologique, cet article se veut être une rencontre - que nous espérons heuristique - entre une sociologie de l'action publique néo-institutionnaliste et la théorie des champs (une rencontre aux allures de réconciliation - du moins intellectuelle - entre des fractions de la science politique française). Amorcée ailleurs (Roger, 2010 ; Dubois, 2014), cette rencontre - qui vise à étudier de près les relations qui se nouent à l'intérieur et entre les champs - permet de mettre en lumière la manière dont les moments de contingence qui émergent dans chaque champ sont susceptibles de nourrir une concordance entre les projets politiques de certains acteurs. Celle-ci, générant un rapport de force dominant, alimente le changement institutionnel (ou bien son écrasement par les partisans de la reproduction). Ici, l'attention portée aux contraintes structurales - lesquelles donnaient naissance à un moment critique - a permis non pas de saisir la crise comme une variable exogène à l'action mais comme constitutive des conditions au sein desquelles celle-ci se déployait. Le moment critique que constituait la crise du Mediator favorisait un alignement des luttes que menaient des agents appartenant à des champs distincts, ces derniers - tentant d'améliorer leurs positions respectives - ayant bénéfice à tirer de l'accentuation de ladite crise. C'est donc en combinant l'analyse des conditions structurales au sein desquelles se développent les mobilisations pour changer ou reproduire l'action publique qu'une sociologie véritablement politique de l'action de l'État est susceptible de se déployer.

\section{Matthieu Ansaloni \\ Institut national de la recherche agronomique (AGIR) matthieuansaloni@yahoo.fr}

Andy Smith

Université de Bordeaux (Centre Émile Durkheim) a.smith@sciencespobordeaux.fr

\section{Bibliographie}

AlAm, T. (2010), "Les mises en forme savante d'un mythe d'action publique: la sécurité sanitaire", Genèses, 78, p. 48-66.

Ansaloni, M., Pariente, A., Smith, A. (2017), "Beyond the Illusion of Drug Agency Independence. An Inter-field Analysis of the French Case ", Critical
Policy Studies, 7 avril 2017 [doi.org/10.1080/ 19460171.2017.1314220].

BÉgaud, B., Dangoumau, J. (2000), "Pharmacoépidémiologie: définition, problèmes, méthodologie ", Thérapie, 55, p. 113-117.

53. Un appauvrissement qui n'a pas été compensé par la création d'équipes de scientifiques propres à l'agence.

54. L'expression est de Champagne (2016). Nous remercions un évaluateur qui a soulevé cette piste, à nos yeux prometteuse. 
Benamouzig, D., Besançon, J. (2005), «Administrer un monde incertain: les nouvelles bureaucraties techniques. Le cas des agences sanitaires ", Sociologie du travail, 47 (3), p. 301-322.

Benamouzig, D., Besançon, J. (2008), «Les agences ", dans BORRAZ, O., GUIRAUdon, V. (dir.), Politiques publiques. La France dans la gouvernance européenne, Paris, Presses de Sciences Po, p. 309-336.

Benamouzig, D., Pierru, F. (2011), " Le professionnel et le "système": l'intégration institutionnelle du monde médical ", Sociologie du travail, 53 (3), p. 293-348.

Bergeron, H., Nathanson, C. (2012), "Construction of a Policy Arena: The Case of Public Health in France ", Journal Health Politics, Policy and Law, 37 (1), p. 5-36.

Besançon, J. (2010), L'Institutionnalisation de l'agence française de sécurité sanitaire des aliments comme organisation-frontière. Bureaucratisation de l'expertise et régulation des risques alimentaires, Paris, Institut d'études politiques.

Bourdieu, P. (1984), Homo academicus, Paris, Minuit.

BouRdieu, P. (1994), Raisons pratiques. Sur la théorie de l'action, Paris, Seuil.

Bourdieu, P. (2000), Les Structures sociales de l'économie, Paris, Raisons d'agir.

Bourdieu, P. (2012), Sur l'État. Cours au collège de France, Paris, Raisons d'agir.

BOYER, R. (2003), "L'anthropologie économique de Pierre Bourdieu ", Actes de la recherche en sciences sociales, 150, p. 65-78.

Buton, F. (2006), « De l'expertise scientifique à l'intelligence épidémiologique: l'activité de veille sanitaire », Genèses, 65, p. 71-91.

Buton F., Pierru, F. (2012), "Instituer la police des risques sanitaires. Mise en circulation de l'épidémiologie appliquée et agencification de l'État sanitaire ", Gouvernement et action publique, 4 (4), p. 67-90.

Callon, M., Lascoumes, P., Barthe, Y. (2001), Agir dans un monde incertain, Paris, La Découverte.

Carpenter, D. (2011), Reputation and Power: Organizational Image and Pharmaceutical Regulation at FDA, Princeton (N. J.), Princeton University Press.

Champagne, P. (2016), La Double dépendance. Sur le journalisme, Paris, Raisons d'agir.

Dangoumau, J. (2006), Pharmacologie générale, Bordeaux, Université Victor Segalen.

Debarge, O. (2012), "Les désaccords entre la France et l'Union européenne relatifs à la suspension de certains médicaments: simple discordance ou mal profond ? ", Revue générale de droit médical, 43, p. 319-329.

DoBry, M. (1983), "Mobilisations multisectorielles et dynamiques des crises politiques ", Revue française de sociologie, 24 (3), p. 395-419.

Dobry, M. (1986), Sociologie des crises politiques, Paris, Presses de Sciences Po.

DuBoıs, V. (2014), "L'action de l'État, produit et enjeu des rapports entre espaces sociaux ", Actes de la recherche en sciences sociales, 201-202, p. 11-25.

Fligstein, N., McAdam, D. (2012), A Theory of Fields, Oxford, Oxford University Press.

GILARDI, F. (2005), "The Formal Independence of Regulators: A Comparison of 17 Countries and 7 Sectors", Swiss Political Science Review, 11 (4), p. 139-167.

Gilardi, F., Maggettı, M. (2010), "The Independence of Regulatory Authorities ", in LevI-FAUR, D. (ed.), Handbook of Regulation, Cheltenham, Edward Elgar, p. 201-214.

Granjou, C., Barbier, M. (2004), "Une nouvelle transparence dans la communication des savoirs et des incertitudes scientifiques? Le Rôle des agences", colloque "Sciences, médias et sociétés ", Lyon, 15-17 juin.

HanRetTy, C., Koop, C. (2012), "Measuring the Formal Independence of Regulatory Agencies", Journal of European Public Policies, $19(2)$, p. 198-216.

HanRetty, C., Koop, C. (2013), "Shall the Law Set them Free? The Formal and Actual Independence of Regulatory Agencies ", Regulation and Governance, 7 (2), p. 195-214.

Hassenteufel, P. (1997), Les Médecins contre l'État, Paris, Presses de Sciences Po.

Hassenteufel, P., Bachir, M., Bussat, V., Genieys, W., Martin, C., Serre, M. (1999), L'Émergence d'une nouvelle "élite du welfare"? Sociologie des sommets de l'État en interaction. Le cas des politiques de protection maladie en matière de prestations familiales (1981-1997), Paris, MIRE.

Hauray, B. (2005), L'Europe du médicament. Politique, expertise, intérêts privés, Paris, Presses de Sciences Po.

Hillaire-Buys D., Faillie, J.-L., Petit, P., MontasTRUC, J.-L. (2013), « Scientific Evidence and Controversies about Pioglitazone and Bladder Cancer: Which Lessons Can Be Drawn?", Drug Safety, 36 (9), p. 693-707.

Hitlaire-Buys, D., Faillie, J.-L., Montastruc, J.-L., PetIT, P. (2010), "Stay Vigilant: A Glitazone (Pioglitazone) Can Hide a Glitazar! ", European Journal of Clinical Pharmacology, 68 (12), p. 1681-1683. 
Hillaire-Buys, D., Montastruc, J.-L. (2011), "Pioglitazone and Bladder Cancer ", The Lancet, 378, p. $1543-1544$.

ItÇAINA, J., Roger, A., Smith, A. (2016), Varietals of Capitalism. A Political Economy of the Changing Wine Industry, Ithaca (N. Y.), Cornell University Press.

Jones, J., KINGERY, E. (2014), " History of Pharmacovigilance", in Andrews, E., Moore, N. (eds), Mann's Pharmacovigilance, New York (N. Y.), WileyBlackwell, p. 11-24.

LE GalÈs, P. (2014), "Recomposition de l'État: changement d'échelle, normes extérieures, nouvelles organisations ", dans LE GALĖs, P., VeZINAT, N. (dir.), L'État recomposé, Paris, PUF, p. 5-30.

LeVI-Faur, D. (2005), « The Global Diffusion of Regulatory Capitalism ", The Annals of the American Academy of Political and Social Science, 598 (12), p. 12-32.

Lewis, J.-D., Ferrara, A., Peng, T., Hedderson, M., BILKER, W.-B., QuesenBerRY, C.-P. (2011), « Risk of Bladder Cancer among Diabetic Patients Treated with Pioglitazone: Interim Report of a Longitudinal Cohort Study ", Diabetes Care, 34, p. 916-922.

Maggetti, M., Verhoest, K. (2014), "Les aspects inexplorés de l'autonomie bureaucratique : état des lieux et perspectives", Revue internationale des sciences administratives, 80 (2), p. 243-260.

Majone, G. (1997), La Communauté européenne: un État-régulateur, Paris, Montchrestien.

MARCHETtI, D. (2010), Quand la santé devient médiatique, Grenoble, Presses universitaires de Grenoble.

Moran, M. (2002), "Review Article: Understanding the Regulatory State", British Journal of Political Science, 32, p. 391-413.

Morelle, A. (1996), La Défaite de la santé publique, Paris, Flammarion.
Neveu, É. (2013), "Les sciences sociales doiventelles accumuler les capitaux ? ", Revue française de science politique, 63 (2), p. 337-358.

Noize, P., Saver, M., Bruneval, P., Moreau, M., Pathak, A., Bagheri, H., Montastruc, J.-L. (2006), "Valvular Heart Disease in a Patient Taking Benfluorex", Fundamental and Clinical Pharmacology, 20 (6), p. 572-578.

Pichetti, S., Sermet, C., Ven der Erf, S. (2013), « La diffusion des nouveaux antidiabétiques : une comparaison internationale ", Questions d'économie de la santé, 187, p. 1-8.

Roger, A. (2010), "Constructions savantes et légitimation des politiques européennes. La circulation des savoirs sur la vigne et le vin ", Revue française de science politique, 60 (6), p. 1091-1113.

Rose, N., Miller, P. (1992), " Political Power beyond the State: Problematics of Government ", The British Journal of Sociology, 43 (2), p. 179-205.

SmITH, A. (2013), "Transferts institutionnels et politiques de concurrence. Les cas communautaire, britannique et français ", Gouvernement et action publique, 3 (3), p. 415-440.

Sommet, A., Bagheri, H., Montastruc, J.-L. (2007), "De la pharmacovigilance à la gestion des risques ", La Lettre du pharmacologue, 21 (1-2), p. 26-31.

TABUTEAU, D. (1994), La Sécurité sanitaire, BoulogneBillancourt, Berger-Levrault.

TAButeau, D. (1996), "Les pouvoirs publics et le risque sanitaire", Revue française des affaires sociales, 50 (2), p. 29-40.

Tabuteau, D. (2010), "L'expert et la décision en santé publique ", Les Tribunes de la santé, 27 (2), p. 33-48.

URFALINO, P. (2001), «L'autorisation de mise sur le marché du médicament : une décision administrative à la fois sanitaire et économique ", Revue française des affaires sociales, 4 (4), p. 85-90. 